\title{
INTERMITTENCY IN THE PHOTOSPHERE AND CORONA ABOVE AN ACTIVE REGION
}

\author{
Valentyna Abramenko, Vasyl Yurchyshyn, and Haimin Wang ${ }^{1}$ \\ Big Bear Solar Observatory, Big Bear City, CA 92314 \\ Received 2008 January 22; accepted 2008 March 16
}

\begin{abstract}
Recent studies have demonstrated without doubt that the magnetic field in the photosphere and corona is an intermittent structure, opening new views of the underlying physics. In particular, such problems as the existence in the corona of localized areas with extremely strong resistivity (required to explain magnetic reconnection at all scales) and the interchange between small and large scales (required in the study of photospheric-coronal coupling), to name a few, can be easily captured by the concept of intermittency. This study focuses on simultaneous time variations of intermittency properties derived in the photosphere, chromosphere, and corona. We analyze data for NOAA Active Region 10930 acquired between 2006 December 8, 12:00 UT, and December 13, 18:45 UT. Photospheric intermittency is inferred from Hinode magnetic field measurements, while intermittency in the transition region and corona is derived from Nobeyama $9 \mathrm{GHz}$ radio polarization measurements and high-cadence Hinode XRT (thinBe) data, as well as GOES 1-8 $\AA$ flux. The photospheric dynamics and its possible relationship with the intermittency variations are also analyzed by calculating the kinetic vorticity. In this case study, we find the following chain of events: The intermittency of the photospheric magnetic field peaked after the specific kinetic vorticity of plasma flows in the active region reached its maximum ( $4 \mathrm{hr}$ time delay). In turn, a gradual increase of coronal intermittency occurred after the peak of the photospheric intermittency. The time delay between the peak of photospheric intermittency and the occurrence of the first strong (X3.4) flare was approximately 1.3 days. Our analysis seems to suggest that the enhancement of intermittency/complexity first occurs in the photosphere and is later transported toward the corona.
\end{abstract}

Subject headings: Sun: corona - Sun: magnetic fields — Sun: photosphere

Online material: color figures

\section{INTRODUCTION}

It is a widespread view that the eruptive processes of energy release in the solar corona seem to be independent of the dynamics observed in the moderately varying photosphere. This view is partially based on the fact that no one-to-one correlation has been observed between coronal and photospheric dynamics. For example, numerous attempts to find any persistent preflare changes in the photosphere have yet to lead to any solid conclusions, although recent efforts to detect flare-related changes in photospheric magnetic fields have been more promising (Spirock et al. 2002; Wang et al. 2004; Sudol \& Harvey 2005; Wang 2006). More than a dozen publications by different groups have reported that persistent, abrupt changes in the photospheric magnetic flux occur in association with X-class flares. The most plausible explanation for the observed phenomena seems to be flare-related changes of the inclination of field lines rooted in the photosphere. If this is the case (future analysis of high-cadence vector magnetograms could be helpful), then we probably are dealing with feedback to the photosphere from reorganizing coronal fields.

The question whether there is any forward reaction of the photosphere to the corona is still open, however. There seems to be some acceptance that a statistical relationship may exist between the conditions in the photosphere and corona: for a large enough ensemble of active regions, a good correlation can be found between photospheric magnetic parameters and coronal phenomena (Fisher et al. 1998; Falconer et al. 2003; Schrijver et al. 2004; Abramenko 2005b; McAteer et al. 2005; Schrijver \& Title 2005; Abramenko et al. 2006; Jing et al. 2006; Tan et al. 2007; Leka \&

\footnotetext{
${ }^{1}$ Center for Solar-Terrestrial Research, New Jersey Institute of Technology, Newark, NJ 07102.
}

Barnes 2007 and references therein; Georgoulis \& Rust 2007; Conlon et al. 2008). From the theoretical standpoint, consensus seems to have been reached that the ultimate source of energy for coronal energy releases is the photospheric and subphotospheric motions of magnetized plasma. Various mechanisms have been suggested for the energy transport toward the corona, which can and should be observationally validated (for reviews of coronal heating mechanisms, see, e.g., Malara \& Velli 1994; Schrijver \& Title 2005; Klimchuk 2006). However, various statistical correlations do not seem to be adequate any longer, and timing characteristics are required.

Indeed, when we address issues of the coupling between any two systems, a question of vital importance immediately arises: do events that occur in one system persistently precede or follow events in another system? If the answer is "yes," then what is the characteristic time delay between a pair of related events? A shorter delay suggests a closer and more intimate coupling, while long time delays may indicate a less straightforward and more complex relationship. In particular, in such dynamical systems as magnetized turbulent plasma the interplay between scales may influence the delay time. In this case it is advantageous to adopt an intermittency approach, a technique that allows one to capture interactions between various scales, in the best way possible as of today. In the present study, we undertake an attempt to detect the delay intervals between key moments of the intermittency behavior in the photosphere and corona.

\section{DATA ANALYSIS}

High spatial and temporal resolution measurements of the photospheric magnetic field and solar corona performed recently by a set of Hinode instruments (Kosugi et al. 2007) provide us with a 

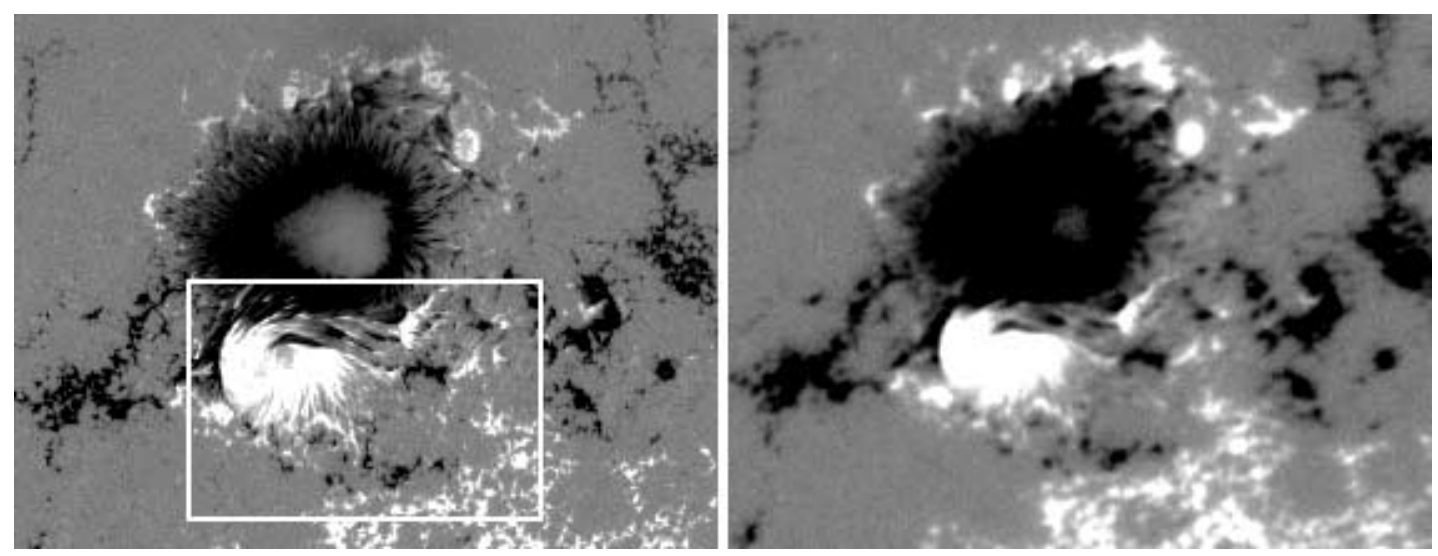

FIG. 1.-Line-of-sight magnetograms of NOAA AR 10930 recorded on 2006 December 12 at 00:48 UT by the Hinode SOT/FG (left) and SOHO MDI (right) instruments. The MDI high-resolution magnetogram is scaled from -300 to $300 \mathrm{G}$, the SOT/FG from -300 to $300 \mathrm{DN}$. The white rectangle encloses the area used for calculations of the intermittency index $\kappa$ and the squared kinetic vorticity $\left\langle\omega^{2}\right\rangle$.

unique opportunity to simultaneously estimate the degrees of intermittency in the photosphere and corona and to track their variations in time. The Hinode Space Optical Telescope's "Filtergraph" camera (SOT/FG) is designed to produce filter-based vector magnetograms with high spatial and temporal resolution. Strictly speaking, these images only represent measurements of the Stokes $V$ polarization parameter at a single wavelength, so information on the magnetic field intensity is not available in these data. However, the data do bear information about the magnetic structuring, with an unprecedented spatial resolution of $0.16^{\prime \prime}$. These uninterrupted measurements are taken with a high time cadence of 2 minutes and cover several days of observations of an active region. An example of an SOT/FG Hinode magnetogram for NOAA Active Region 10930 is shown in the left panel of Figure 1, while the right panel shows a simultaneous Solar and Heliospheric Observatory (SOHO) Michelson Doppler Imager (MDI) high-resolution magnetogram of the same area on the Sun. As far as spatial resolution is concerned, the advantage of the SOT/FG data is obvious.
In this paper, we analyze properties of photospheric intermittency by utilizing (1) a technique that relates the slope of a "flatness function" to the degree of intermittency, and (2) calculations of the kinetic vorticity in the photosphere. Three independent data sets are used to calculate the intermittency in the corona and chromosphere: $\mathrm{X}$-ray emission records from the Hinode X-Ray Telescope (XRT) and GOES, as well as Nobeyama $9.4 \mathrm{GHz}$ polarization flux.

We analyze NOAA AR 10930, observed by Hinode in 2006 December. During its passage across the solar disk, this active region displayed at least two periods of enhanced activity, separated by a long interval of relative quietness. The first flaring interval lasted from 2006 December 4 to December 7, when the active region was very close to the east limb. As evidenced by a series of MDI full-disk magnetograms, at the end of this activity period the magnetic complexity in the active region was nearly exhausted and a new period of complexity gain was setting in. This second activity period was accompanied by the gradual emergence of a fast-rotating sunspot of positive $(\mathrm{N})$ polarity located in close proximity to the main, negative-polarity sunspot. The emergence

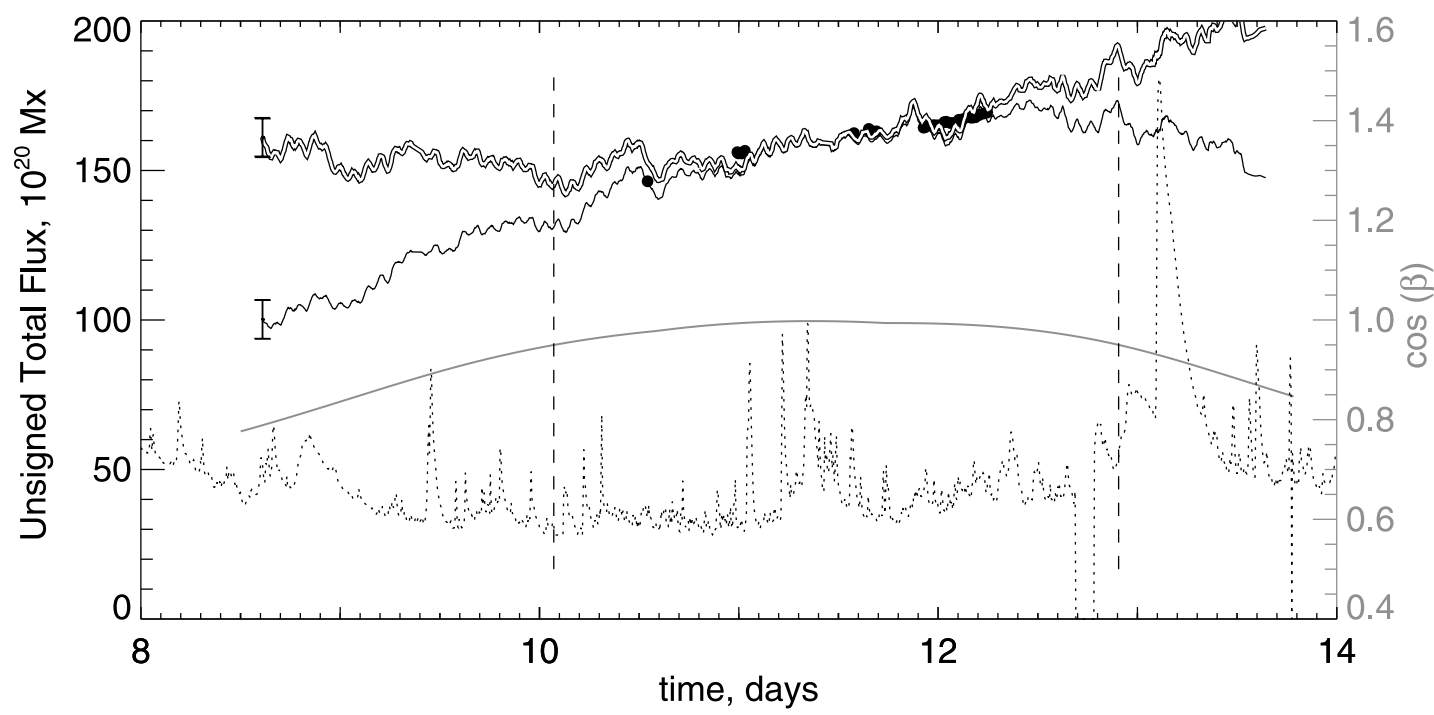

FIG. 2.- Time variation of the unsigned magnetic flux in NOAA AR 10930 calculated from SOT/FG magnetograms (arbitrary units): thin solid line, unsigned

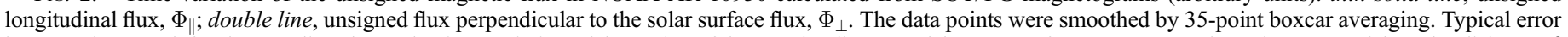

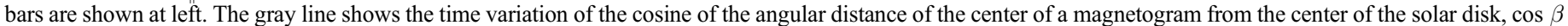

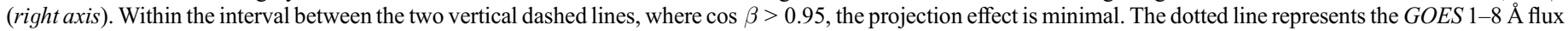

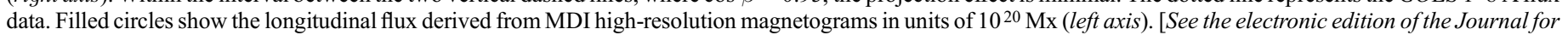
a color version of this figure.] 

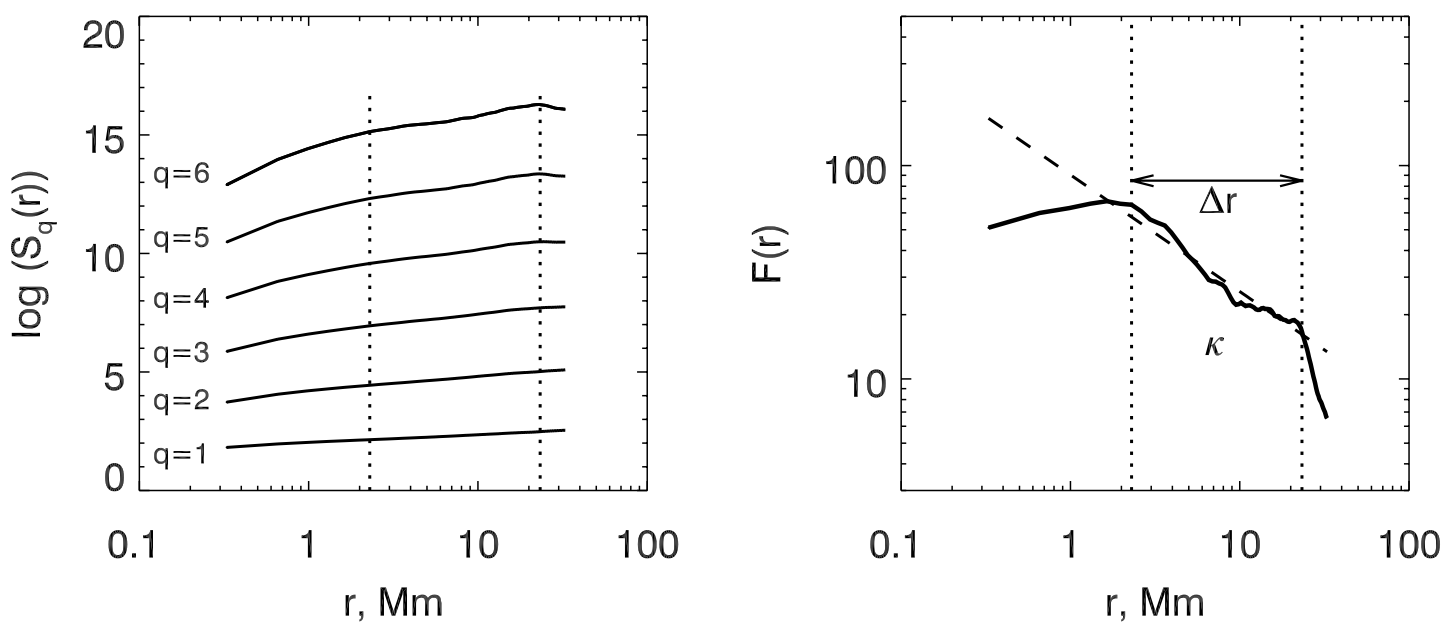

FIG. 3.-Left, structure functions $S_{q}(r)$ (eq. [1]) obtained from the SOT/FG magnetogram shown in Fig. 1 (the white rectangle there); right, flatness function $F(r$ ) derived from the structure functions (eq. [2]). Vertical dotted lines mark the intermittency interval $\Delta r$ in which the flatness grows according to a power law when $r$ decreases. The interval $\Delta r$ is also marked in the left panel. The intermittency index $\kappa$ is the slope of $F(r)$ determined within $\Delta r$ : the flatness function steepens as the magnetic field becomes more intermittent. For the magnetogram under consideration, $\kappa=0.55 \pm 0.05$ was determined from a linear regression (dashed line) within the range $\Delta r=2.3-23 \mathrm{Mm}$.

and rotation of the positive sunspot ceased with the occurrence of two powerful X-class flares on 2006 December 13 and 14. The time period between December 8, 12:00 UT, and December 13, 18:45 UT, was chosen to study how time variations of magnetic complexity in the photosphere and corona are related.

We analyzed $1718 \mathrm{SOT} / \mathrm{FG} 2 \times 2$ rebinned magnetograms taken with a time cadence of 4 minutes. During the analyzed time interval, the active region moved across the solar disk from a longitude of $37^{\circ}$ east to $32^{\circ}$ west, so that the influence of the projection effect should be considered. We integrated the longitudinal flux density, $\mid B \|$, over the entire number $N$ of pixels, of size $\Delta S=0.32^{\prime \prime} \times 0.32^{\prime \prime}$, occupied by the active region. We thus obtained the longitudinal total unsigned flux, $\Phi_{\|}$, which is plotted in Figure 2 (thin solid line). The assumption that the magnetic field in the photosphere is predominantly vertical to the solar surface offers the possibility to estimate the magnetic flux perpendicular to the solar surface, $\Phi_{\perp}$ (Murray 1992; Hagenaar 2001). For each magnetogram, we calculated the cosine of the angular distance from the center of the magnetogram to the center of the solar disk, $\cos \beta$ (Fig. 2, gray line). The perpendicular flux density thus can be estimated as $B_{\|} / \cos \beta$ and the deprojected pixel area is $\Delta S / \cos \beta$, which results in an estimated magnitude for the perpendicular unsigned flux of $\Phi_{\perp}=\Phi_{\|} / \cos ^{2} \beta$ (Fig. 2, double line). During the time range for which $\cos \beta>0.95$ (between the dashed vertical lines), the perpendicular and longitudinal fluxes differ by less than $10 \%$. We therefore accept that within this time range our data and results are essentially free from the projection effect.

\section{MEASURE OF INTERMITTENCY}

Intermittency manifests itself in both the spatial (two- or threedimensional) and temporal (one-dimensional) domains. In the spatial domain, intermittency implies a tendency of the magnetic field to concentrate into small-scale flux tubes of high intensity, surrounded by extended areas of much weaker field. This tendency becomes more pronounced as the spatial resolution of the data increases. In the temporal domain, intermittency is evidenced through a burstlike behavior of events. Studies of intermittency in both the spatial and temporal domains can be conducted with the same techniques, such as the structure function approach (see, e.g., Frisch 1995).
Structure functions, defined as the statistical moments of the increments of a field, are a useful tool for exploring intermittency (Stolovitzky \& Sreenivasan 1993; Frisch 1995; Consolini et al. 1999; Abramenko et al. 2002, 2003; Abramenko 2005a; Buchlin et al. 2006; Uritsky et al. 2007). In our case the analyzed field is the line-of-sight component, $B_{\|}$, of the photospheric magnetic field, so that the structure function can be defined as

$$
S_{q}(r)=\left\langle\left|B_{\|}(\boldsymbol{x}+\boldsymbol{r})-B_{\|}(\boldsymbol{x})\right|^{q}\right\rangle,
$$

where $\boldsymbol{x}$ is a given pixel on a magnetogram, $\boldsymbol{r}$ is a separation vector, and $q$ is the order of the statistical moment, which takes real values. Angle brackets denote an average over the entire magnetogram.

Generally, the ratio of the fourth-order structure function to the square of the second-order structure function determines the flatness function. However, in the case of intermittency analysis, Frisch (1995) suggested using even higher statistical moments and to calculate the (hyper-) flatness as the ratio of the sixth moment to the cube of the second moment:

$$
F(r)=S_{6}(r) /\left[S_{2}(r)\right]^{3} \sim r^{-\kappa} .
$$

For nonintermittent structures, the flatness does not depend on the spatial scale $r$. On the contrary, for an intermittent structure the flatness grows as a power law when the scale $r$ decreases (Frisch 1995; Abramenko 2005a). The intermittency index, $\kappa$, which is determined as the slope of the flatness function within a spatial range of linearity $\Delta r$ (see Fig. 3) increases when the intermittency is higher.

We applied the above technique to analyze the solar intermittency in both the spatial and temporal domains. To process one-dimensional time series, we modified our two-dimensional flatness function code based on equations (1) and (2) by replacing the spatial scale with the timescale, $\tau$, and the magnetic field, $B_{\|}$, with a time series of coronal measurements.

First we determined the flatness functions and intermittency indices for all selected SOT/FG magnetograms. To avoid possible contamination of the results due to the saturation effect inside the main sunspot and the vast area of weak fields around it, only the fields enclosed by the white rectangle in Figure 1 (the fast-rotating spot and flare site) were used in the calculations. 


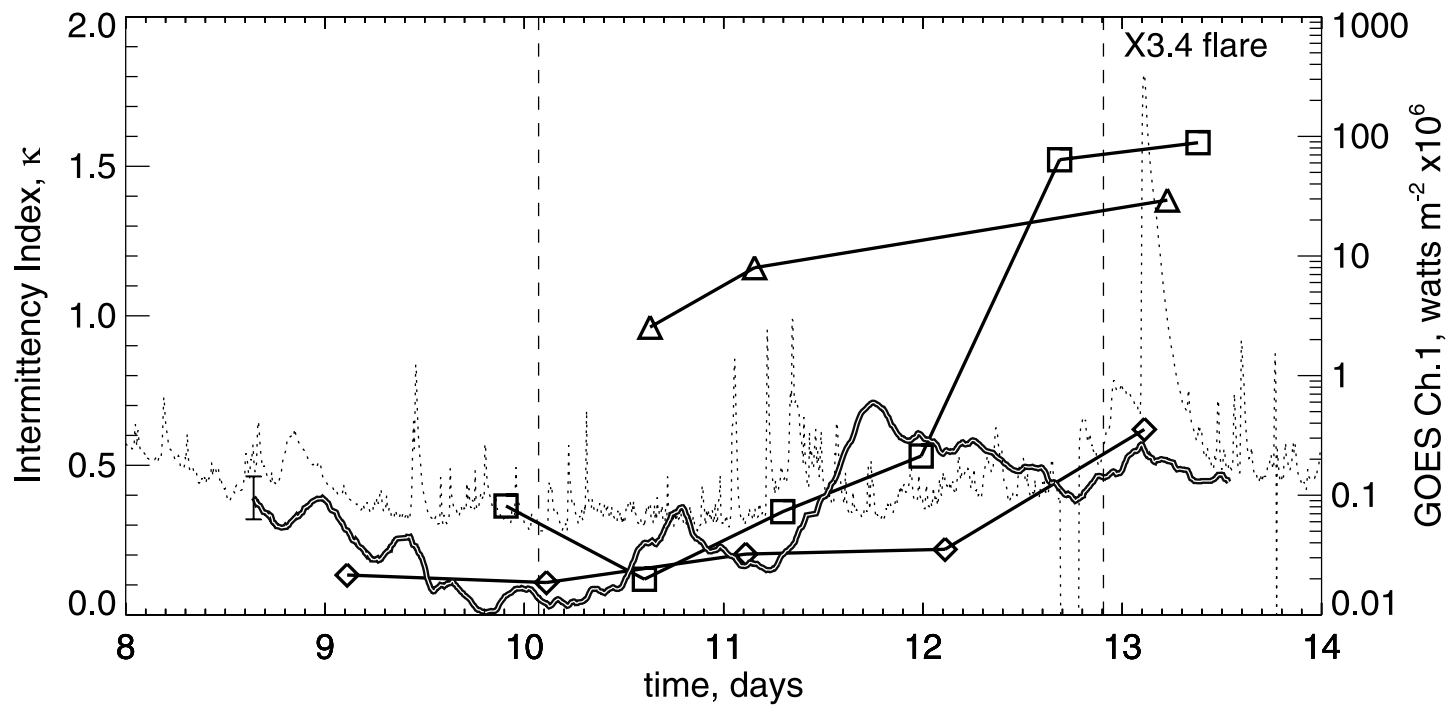

FIG. 4.-Time variation of the intermittency index, $\kappa$, determined from (1) photospheric magnetograms (double line; the data points were smoothed with 35-point boxcar averaging, and a typical error bar is shown at left), (2) XRT data (triangles), (3) Nobeyama radio data (diamonds), and (4) GOES flux (squares). For the last three cases, the error bars are smaller than the symbol size. Other notations are the same as in Fig. 2. [See the electronic edition of the Journal for a color version of this figure.]

The range $\Delta r=2.3-23 \mathrm{Mm}$, as shown in Figure 3, was adopted for all the magnetograms.

The double line in Figure 4 shows the time profile of $\kappa$ in the photosphere. The intermittency index peaks on December 11 at about 18:00 UT (day 11.75), which is approximately 1.3 days before the X3.4 flare. This peak is located inside the projectionfree time interval (between the vertical dashed lines), and its magnitude significantly exceeds the error bar, which allows us to consider it a real change in the complexity of the photospheric magnetic field.

Second, to explore the properties of intermittency in the solar chromosphere and corona, we utilized time series obtained from various instruments such as the Hinode XRT, GOES, and the Nobeyama Radio Polarimeters. Both hard X-ray and radio fluxes are direct tracers of electrons accelerated in reconnection events. Intermittency analysis based on these data may reveal information about the chromospheric and coronal reconnection dynamics, that is, reorganization in the magnetic field.
We used 1 minute thin-Be filter data taken with the Hinode XRT instrument (Kosugi et al. 2007; Golub et al. 2007). These images were processed with the standard SSW IDL software package, and the XRT flux was calculated by integrating pixel intensities over the active region (Fig. 5). The XRT data for this time interval are not continuous. We analyzed three subsets acquired on days 9.4-10.4, 10.4-11.4, and 13.0-13.7. We denote these the December 10, December 11, and December 13 data sets. Each subset contains about 1000 data points. For each subset, we calculated the flatness function (Fig. 6, left) and the intermittency index $\kappa$.

The data presented in Figure 6 allow us to explain how we select a linear interval, $\Delta r$, for the time series. In each case, a linear range was detected in the middle part of the spectrum. We then extend this range in both directions and recalculate the linear fit and the slope $\kappa$. We continue to do this while the variations in $\kappa$ remain within the standard deviation of the linear fit, which is in most of the cases less than 0.05 .

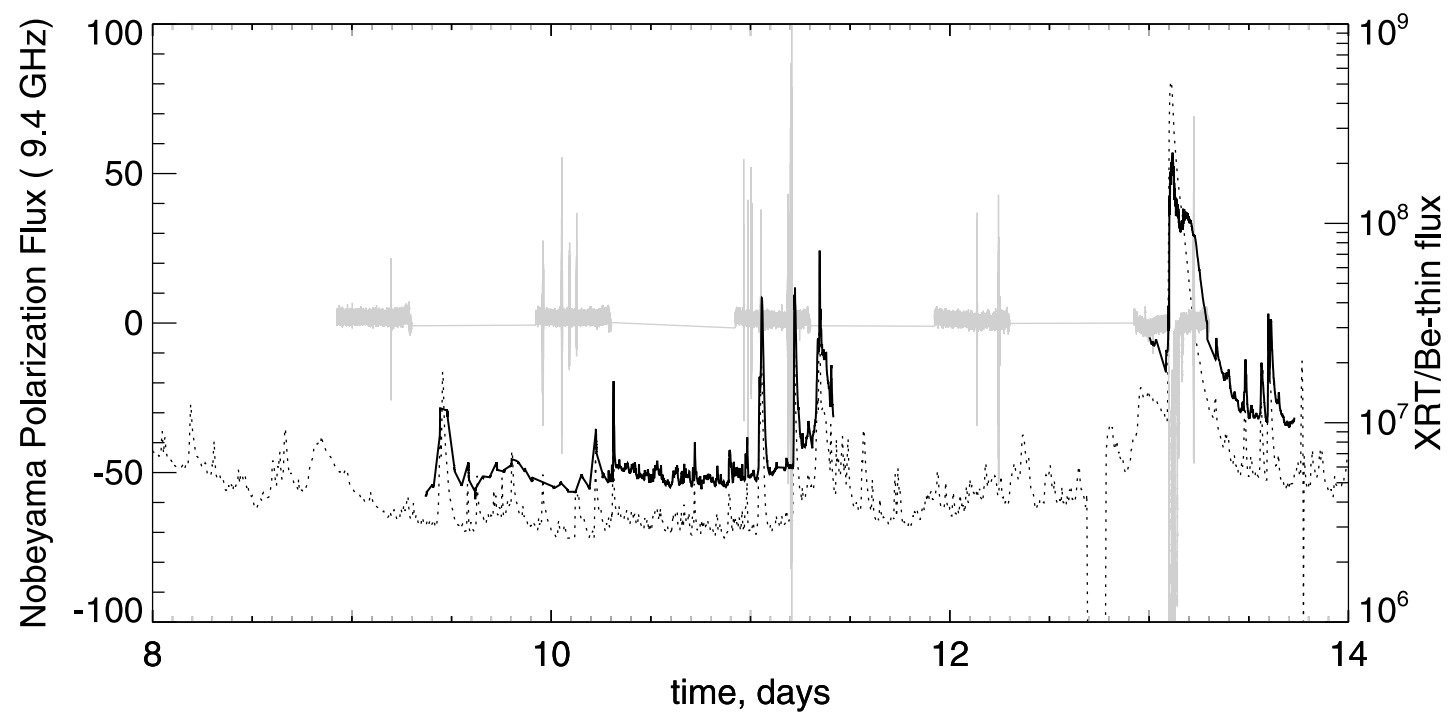

FIG. 5.- Time variation of the Hinode XRT thin-Be flux integrated over the active region area (solid black line) and the radio polarization flux at 9.4 GHz from the Nobeyama Radio Polarimeters (gray line). The dotted line represents the GOES flux. [See the electronic edition of the Journal for a color version of this figure.] 

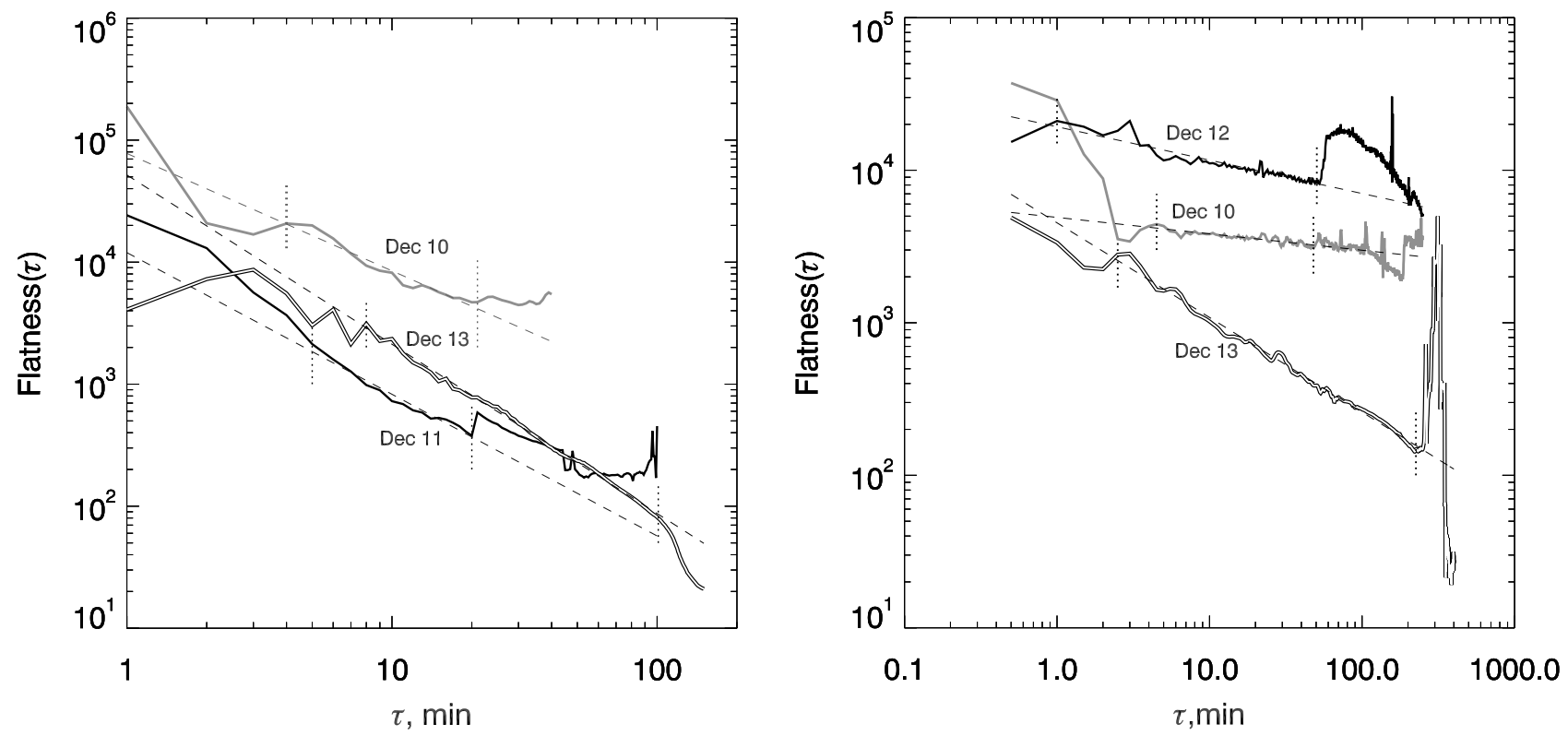

FIG. 6. - Left: Flatness functions calculated for three time series of XRT thin-Be filter flux. For the December 10 set, $\kappa=0.92 \pm 0.05$ within $\Delta r=4-21$ minutes; for

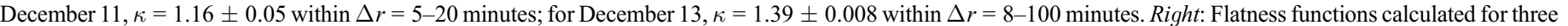

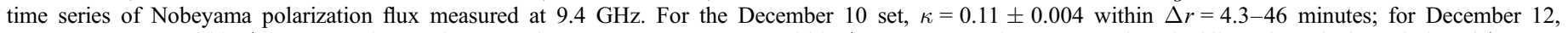

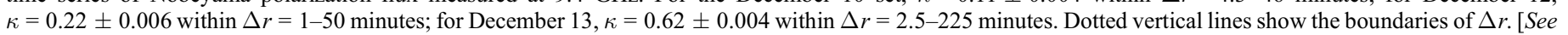
the electronic edition of the Journal for a color version of this figure.]

The polarization flux at $9.4 \mathrm{GHz}$ from the Nobeyama Radio Polarimeters is shown in Figure 5. The radio emission at this frequency is predominantly determined by the gyroresonance process and is largely controlled by the strength and dynamics of the magnetic fields above active regions (Kundu 1965; Aschwanden 2002). We rebinned these $1 \mathrm{~s}$ data so that new time sampling was $30 \mathrm{~s}$ and calculated the flatness function for each observing day, thus obtaining five estimates of $F(\tau)$. Three of them are presented in Figure 6 (right). The corresponding values of $\kappa$ are shown in Figure 4.

As evidenced by both the XRT and Nobeyama data (Fig. 6), the slope of the flatness function gradually steepened from December 10 to 13, implying a gradual increase of intermittency. One more interesting detail can be noted in the behavior of $F(\tau)$, namely, that the large-scale end of the linear interval (i.e., the interval of scales involved in the intermittent process) shifts toward larger scales as $\kappa$ increases (see the functions for December 13). This means that growing intermittency (complexity) involves increasingly longer timescales. Note that similar behavior (involvement of larger spatial scales) was also observed in the case of the photospheric magnetic field.

The same intermittency calculation routines were applied to six 1 day time series of 1 minute GOES 1-8 Å fluxes. In Figure 4, we compare the time variations of the intermittency indices in the photosphere, chromosphere, and corona. The photospheric data show an undulating behavior with a prominent peak on December 11 and a gradual decrease after that, while the coronal and chromospheric indices continue to increase through December 13. Thus, the data seem to suggest that intermittency may first increase in the photosphere and then propagate toward the chromosphere and corona.

What processes in the photosphere and below are responsible for this gain of complexity and intermittency? It is thought that convective and turbulent motions of the magnetized plasma in photospheric and subphotospheric layers could be responsible for increasing complexity (see, e.g., the review by Klimchuk 2006). Statistical comparisons (e.g., Abramenko et al. 2006; Jing et al.
2006; Tan et al. 2007) seem to agree with this assumption. If so, photospheric dynamics could be compared with the intermittency indices, and the attention should be focused on the kinetics of the photospheric magnetic flux tubes.

\section{PHOTOSPHERIC KINETIC VORTICITY}

Hinode SOT/FG Level-0 magnetograms are very well suited for analyzing horizontal displacements of magnetic elements. To measure these displacements and their speed, we utilized the local correlation tracker (LCT) technique (Strous et al. 1996), which was applied to the same set of SOT/FG magnetograms that we used for the intermittency analysis.

The FWHM of the Gaussian tracking window was $9^{\prime \prime} \times 9^{\prime \prime}$. This window size was chosen to provide an optimal trade-off between the noise signal and the spatial resolution of the flow map. A flow map was calculated for each pair of successive images in the data set, based on a 4 minute correlation interval. We estimate that the solar noise (an error signal introduced by the evolution of solar features) is less than $30 \mathrm{~m} \mathrm{~s}^{-1}$. An example flow map derived for the magnetogram in Figure 1 (left) is shown in Figure 7.

For each flow map we then calculated the kinetic vorticity, $\omega$, by using the integral formula

$$
\omega(\boldsymbol{r})=\lim _{s \rightarrow 0} \frac{1}{S} \int_{L} \boldsymbol{v}_{\perp}(\boldsymbol{r}) d l,
$$

where the integration is performed along a contour $L$ enclosing an area $s$ that contains the point $r$. In comparison with the traditional differential technique, our approach appears to be more accurate and offers the possibility to integrate using accurate methods, such as Simpson's rule.

In our code, the area $s$ is represented by the Gaussian tracking window, $s=\Delta x \times \Delta y$. The components of the transverse velocity, $v_{x}(i, j)$ and $v_{y}(i, j)$, are interpolated onto a refined mesh of $\Delta x / 2 \times \Delta y / 2$ pixel size, and then the integration, in the counterclockwise direction along the contour $L=[\Delta x, \Delta y,-\Delta x,-\Delta y]$, 


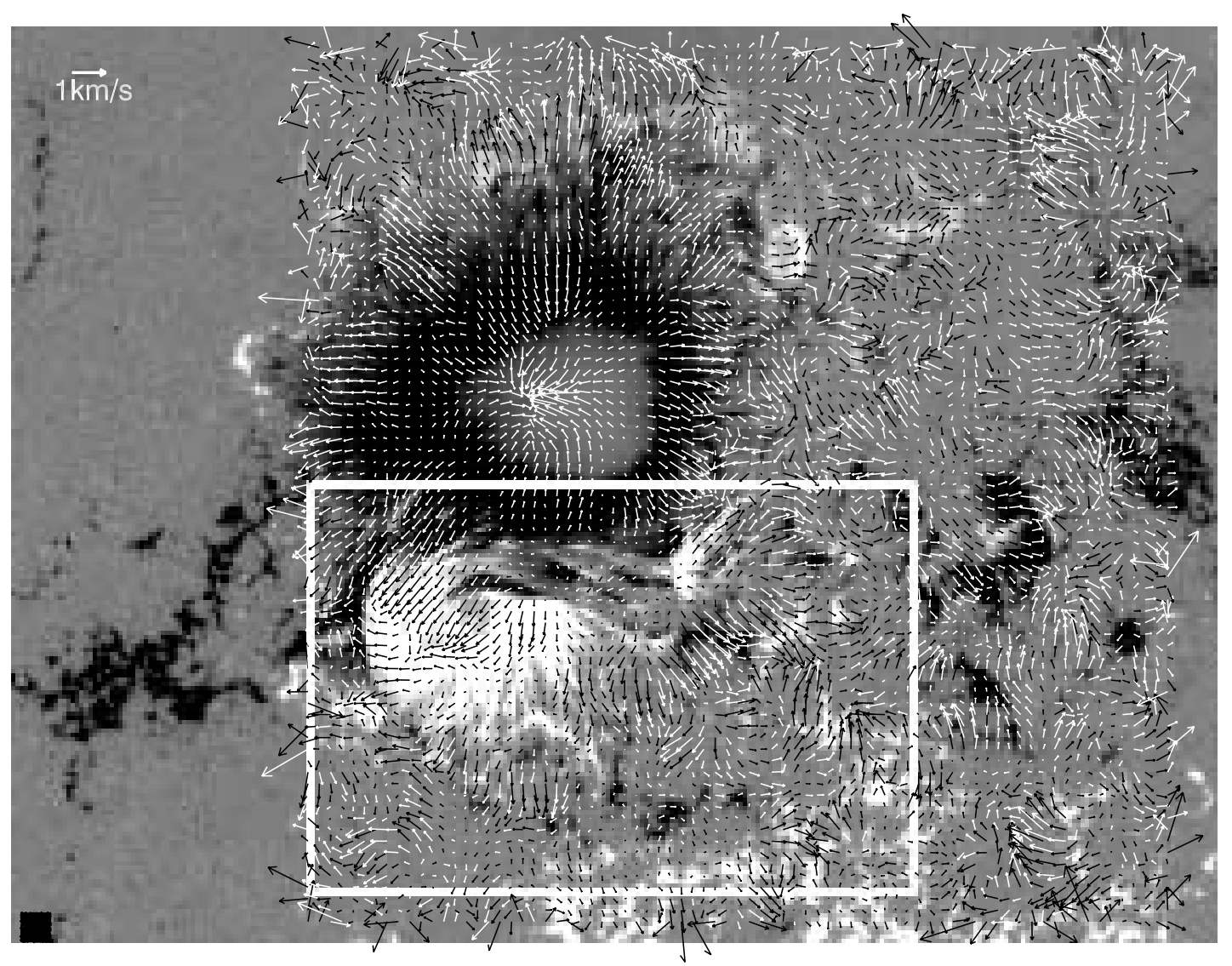

FIG. 7. - Horizontal velocities of magnetic elements derived with the LCT technique for the 2006 December 12, 00:48 UT magnetogram. Notations are the same as in Fig. 1.

which encloses the current point $\boldsymbol{r}(i, j)$, is performed. The integrals along each side of $L$ are calculated with Simpson's formula. For example, the integral along the positive $x$-direction (the bottom side of $L$ ) is

$$
I_{1}=\frac{1}{6} \Delta x\left[v_{x}(m, n)+4 v_{x}(m+1, n)+v_{x}(m+2, n)\right] .
$$

Here the indexes $m$ and $n$ belong to the refined mesh, so that $m=2 i$ and $n=2 j$. The sum of the four integrals divided by $s$ gives us the estimate of the kinetic vorticity at the current point $\boldsymbol{r}(i, j)$.

We note that an analog to the kinetic vorticity is electric current, which can be calculated by replacing the flow field $v_{\perp}$ in equation (3) with the horizontal magnetic field $B_{\perp}$. Having the map of $\omega(\boldsymbol{r})$, we calculated the squared kinetic vorticity averaged over the area, $\left\langle\omega^{2}\right\rangle$. This parameter characterizes the dissipation rate of kinetic energy in the photosphere caused by random motions of the footpoints of magnetic flux tubes.

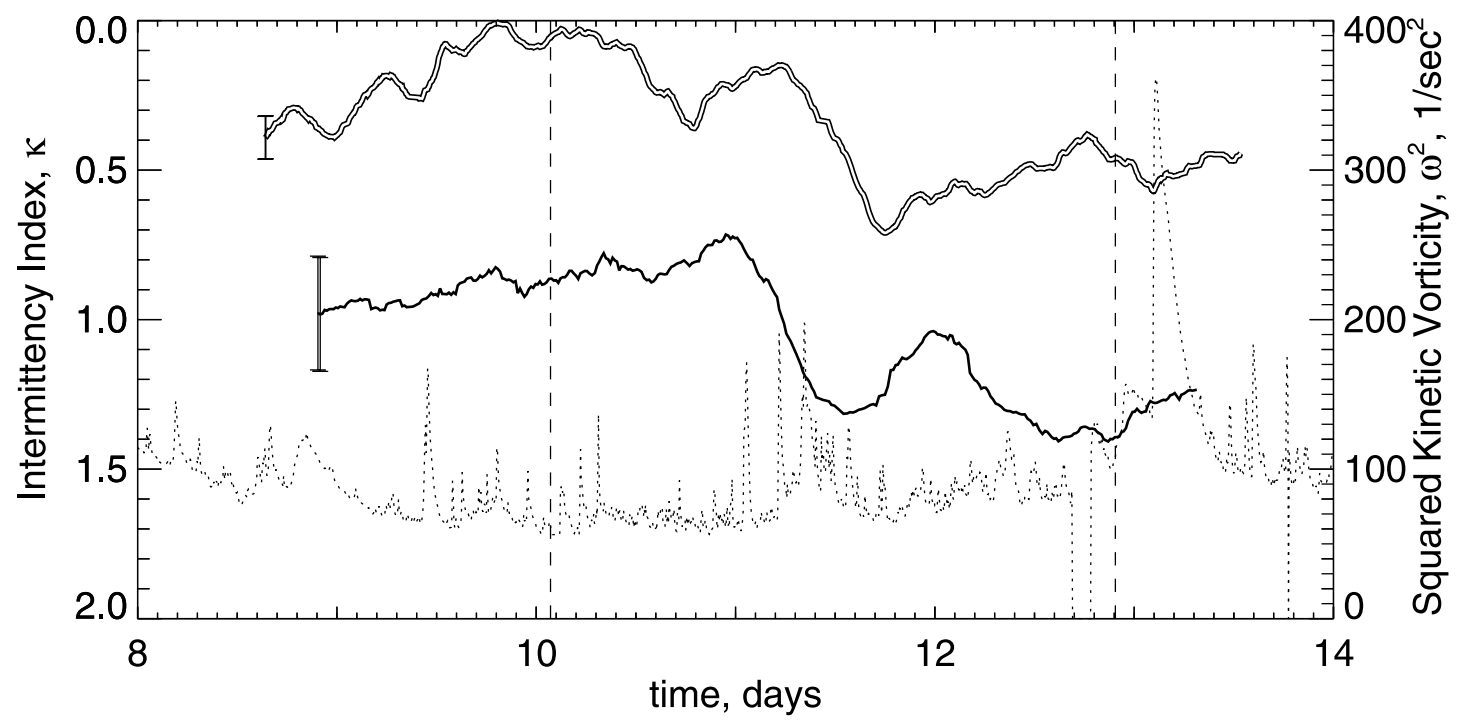

Fig. 8. - Time variation of the area-averaged squared kinetic vorticity, $\left\langle\omega^{2}\right\rangle$ (solid black line), and the intermittency index, $\kappa$, in the photosphere (double line). To facilitate comparison, the left axis is reversed. The dotted line represents the GOES flux. Other notations are the same as in Fig. 2. [See the electronic edition of the Journal for a color version of this figure.] 
In Figure 8, we compare the time variation of $\left\langle\omega^{2}\right\rangle$ and the photospheric intermittency index, $\kappa$. This plot shows that there exists a systematic time lag between the two curves, with the intermittency being delayed. Cross-correlation analysis shows that the delay is approximately $4 \mathrm{hr}$. This indicates that the gain of intermittency in the photosphere is preceded by an enhanced rate of kinetic energy dissipation. In other words, the increase in the kinetic vorticity (or self-rotation of plasma structures) leads to a subsequent increase in the complexity of photospheric magnetic fields.

\section{DISCUSSION AND CONCLUSION}

The case study presented here is devoted to the analysis of the emergence of a rotating sunspot in the close vicinity of a mature spot of opposite polarity. The fact of the rotation was reported by Nightingale et al. (2007) and is considered to be a possible energy source for the X-class flares in this active region. This case demonstrates rather typical evolution of a delta structure: a highly twisted and stressed magnetic flux rope emerges near the preexisting sunspot. The emerging sunspot of opposite polarity "screws" into the active region's magnetic environment. The interaction of the new and old magnetic fluxes is accompanied by a chain of processes: interchange reconnection at the interaction boundary, propagation of the magnetic stress and helicity into the corona, and a gain of complexity in chromospheric and coronal magnetic fields. As a result of magnetic reconnections on a variety of spatial scales, new magnetic connections form. Thus, in the particular case of NOAA AR 10930 Kubo et al. (2007) reported that 1 day before the X3.4 flare, Ca II $\mathrm{H}$ bright loops began appearing near the polarity inversion line. In general, continuous injection of energy and associated magnetic rearrangements may increase the magnetic energy stored in an active region. The result often can be an X-class flare, associated with a coronal mass ejection. In NOAA AR 10930, two powerful X-ray flares were observed on 2006 December 13 and 14.

What can be added to this scenario from the present research? The emergence of the rotating sunspot was associated with undulating variations of the squared kinetic vorticity, $\left\langle\omega^{2}\right\rangle$. The most pronounced peak, observed about 2 days before the X3.4 flare, was followed by an abrupt falloff. Approximately $4 \mathrm{hr}$ after this enhanced activity of photospheric plasma vortices, we observed a peak in the intermittency index of the photospheric magnetic field. Intermittency can be considered a measure of the complexity of the field and implies a tendency of the field to concentrate into extremely strong, widely separated flux tubes (or sheets) with a burstlike behavior of energy releases over time. Intermittency can increase, in particular, as a result of fragmentation and merging, as well as the abrupt intrusion of strong entities. We therefore suggest that the abrupt exhaustion of strong plasma vortices presented more freedom for magnetic flux tubes and thus facilitated fragmentation and merging processes, eventually resulting in a gain of intermittency in the photosphere. A strongly intermittent photospheric magnetic field represents a more stressed magnetic configuration permeated by a multitude of magnetic field discontinuities, which tend to propagate upward because of magnetic tension.

After the photospheric magnetic intermittency peaked, the chromospheric and coronal intermittency continued to increase for at least 1 more day. Approximately 1.3 days after the peak of photospheric intermittency, the first X-class flare was launched. The data allow us to suggest that the magnetic field first became highly intermittent in the photosphere and then intermittency penetrated into the corona, either because of diffusion of magnetic discontinuities or because of waves of various types and their interactions. As a result, a highly critical state in the coronal magnetic configuration was reached, a state that, at any instant, and due to any disturbance, may lead to an eruption.

In the framework of the intermittency concept, the phenomenological scenario for the development of a magnetic structure can be regarded as the evolution of a nonlinear dynamical dissipative system, thus offering a more general insight into the phenomenon. Indeed, a nonlinear dynamical dissipative system is believed to evolve toward its attractor, which is a self-organized criticality (SOC) state. The SOC state is characterized by a not rare occurrence of extremely large fluctuations. These, in turn, result in non-Gaussian distributions of various parameters (recall the power-law distributions of flare energy, flare duration, etc.; see, e.g., Lu \& Hamilton 1991; Charbonneau et al. 2001) and a highly intermittent, or in other words multifractal, organization of the system in the temporal, as well as the spatial, domain. In an SOC state, any perturbation can provoke an eruption of any size, and thus an eruption cannot be predicted in advance.

Of cause, a short-time prediction may be quite possiblerecall that a snow avalanche, for example, can be "predicted" several seconds in advance by sound; however, this is actually a post facto prediction based on the finite speed of avalanche propagation. The same can be said about hard X-ray precursors of $\mathrm{H} \alpha$ flares.

Recently, Leka \& Barnes (2007) came to the conclusion that an individual snapshot of an active region bears little information about the time of an oncoming flare. In our opinion, this inference perfectly agrees with the concept of the evolution of a nonlinear dynamical dissipative system: eruptions cannot be predicted. One can only say that when the system reaches an SOC state, strong eruptions can happen frequently enough, along with a multitude of smaller ones. One of the ways to take a step ahead is to analyze whether a system has reached the SOC state or is still at the stage of accumulating the energy and complexity. How can the SOC state be reached in the corona and what is the role of the photosphere? In the present study, we attempted to tackle these questions. One should keep in mind also that the SOC concept, as any other theory, has its advantages and disadvantages, which are continuously being elaborated (see, e.g., Bélanger et al. 2007).

As for the usefulness of the intermittency/multifractality concept for understanding solar phenomena, it is worth mentioning the long-standing problem of the appearance of low plasma conductivity in the corona, especially during a flare. To explain a solar eruption on the scale of an active region that can last for about 100 minutes, it is necessary to invoke the presence of superstrong electric currents $\left(\sim 10^{10} \mathrm{~A} \mathrm{~km}^{-2}\right)$ inside very thin layers (<100 m; Priest 1982). A fractal concept of coronal magnetic fields can easily meet these requirements. Indeed, a self-similar fractal allows the existence of superthin branches (magnetic sheets or tubes), whereas a percolation state, that is, a large-scale avalanche of an SOC state, implies the formation of superstrong currents at singular branches of the cluster.

The authors would like to thank the anonymous referee for stimulating discussions and suggestions for improvement of the text. This work was supported in part by NASA grants NNG05GN34G and NNX07AT16G and National Science Foundation grant ATM 07-16512. Hinode is a Japanese mission developed and launched by ISAS/JAXA, with NAOJ as domestic partner and NASA and STFC (UK) as international partners. It is operated by these agencies in cooperation with ESA and NSC (Norway). 
Abramenko, V. I. 2005a, Sol. Phys., 228, 29 2005b, ApJ, 629, 1141

Abramenko, V. I., Pevtsov, A. A., \& Romano, P. 2006, ApJ, 646, L81

Abramenko, V. I., Yurchyshyn, V. B., Wang, H., Spirock, T. J., \& Goode, P. R. 2002, ApJ, 577, 487 .2003, ApJ, 597, 1135

Aschwanden, M. J. 2002, Space Sci. Rev., 101, 1

Bélanger, E., Vincent, A., \& Charbonneau, P. 2007, Sol. Phys., 245, 141

Buchlin, E., Vial, J.-C., \& Lemaire, P. 2006, A\&A, 451, 1091

Charbonneau, P., McIntosh, S. W., Liu, H.-L., \& Bogdan, T. J. 2001, Sol. Phys., 203, 321

Conlon, P. A., Gallagher, P. T., McAteer, R. T. J., Ireland, J., Young, C. A., Kestener, P., Hewett, R. J., \& Maguire, K. 2008, Sol. Phys., 248, 297

Consolini, G., Berrilli, F., Pietropaolo, E., Bruno, R., Carbone, V., Bavassano, B., \& Ceppatelli, G. 1999, in Magnetic Fields and Solar Processes, ed. A Wilson (ESA SP-448) (Noordwijk: ESA), 209

Falconer, D. A., Moore, R. L., \& Gary, G. A. 2003, J. Geophys. Res. A, 108(10), No. 1380

Fisher, G. H., Longcope, D. W., Metcalf, T. R., \& Pevtsov, A. A. 1998, ApJ, 508,885

Frisch, U. 1995, Turbulence: The Legacy of A. N. Kolmogorov (Cambridge: Cambridge Univ. Press)

Georgoulis, M. K., \& Rust, D. M. 2007, ApJ, 661, L109

Golub, L., et al. 2007, Sol. Phys., 243, 63

Hagenaar, H. 2001, ApJ, 555, 448

Jing, J., Song, H., Abramenko, V., Tan, C., \& Wang, H. 2006, ApJ, 644, 1273 (erratum 652, 1796)
REFERENCES

Klimchuk, J. A. 2006, Sol. Phys., 234, 41

Kosugi, T., et al. 2007, Sol. Phys., 243, 3

Kubo, M., et al. 2007, PASJ, 59, S779

Kundu, M. R. 1965, Solar Radio Astronomy (New York: Wiley)

Leka, K. D., \& Barnes, G. 2007, ApJ, 656, 1173

Lu, E. T., \& Hamilton, R. J. 1991, ApJ, 380, L89

Malara, F., \& Velli, M. 1994, in IAU Colloq. 144, Solar Coronal Structures, ed.

V. Rušin, P. Heinzel, \& J.-C. Vial (Tatranská Lomnica: VEDA), 443

McAteer, R. T. J., Gallagher, P. T., \& Ireland, J. 2005, ApJ, 631, 628

Murray, N. 1992, ApJ, 401, 386

Nightingale, R. W., Schrijver, C. J., \& Frank, Z. A. 2007, BAAS, 210, No. 94.23

Priest, E. R. 1982, Solar Magneto-Hydrodynamics (Dordrecht: Reidel)

Schrijver, C. J., Sandman, A. W., Aschwanden, M. J., \& DeRosa, M. L. 2004, ApJ, 615, 512

Schrijver, C. J., \& Title, A. M. 2005, ApJ, 619, 1077

Spirock, T. J., Yurchyshyn, V. B., \& Wang, H. 2002, ApJ, 572, 1072

Stolovitzky, G., \& Sreenivasan, K. P. 1993, Phys. Rev. E, 48, R33

Strous, L. H., Scharmer, G., Tarbell, T. D., Title, A. M., \& Zwaan, C. 1996, A\&A, 306, 947

Sudol, J. J., \& Harvey, J. W. 2005, ApJ, 635, 647

Tan, C., Jing, J., Abramenko, V. I., Pevtsov, A. A., Song, H., Park, S.-H., \& Wang, H. 2007, ApJ, 665, 1460

Uritsky, V. M., Paczuski, M., Davila, J. M., \& Jones, S. I. 2007, Phys. Rev. Lett., 99, No. 025001

Wang, H. 2006, ApJ, 649, 490

Wang, H., Liu, C., Qiu, J., Deng, N., Goode, P. R., \& Denker, C. 2004, ApJ, 601, L195 